\title{
NO SYSTEM OF UNCOUNTABLE RANK IS PURELY SIMPLE
}

\author{
FRANK OKOH
}

\begin{abstract}
A pair of complex vector spaces $(V, W)$ is a system if and only if there is a $C$-bilinear map $\mathbf{C}^{2} \times V$ to $W$. The category of systems is equivalent to the category of modules over a certain subring of the ring of $3 \times 3$ matrices over the complex numbers, and so module-theoretic concepts make sense for systems. $A$ system is purely simple if it has no proper pure subsystem. Recently it has been shown that for every positive integer $n$, there exists a purely simple system of rank $n$ but no system of rank greater than the cardinality of the continuum is purely simple. In this paper it is shown that no system of rank greater than $\aleph_{0}$ is purely simple. Necessary and sufficient conditions for a system of rank $\aleph_{0}$ to be purely simple are also given.
\end{abstract}

We shall assume familiarity with the notations and terminology in [3].

LEMMA. Let $(V, W)$ be a nonzero torsion-free system containing no nonzero pure subsystem of finite rank. Then $(V, W)$ contains an ascending sequence of subsystems

$$
\left(V_{1}, W_{1}\right) \subset\left(V_{2}, W_{2}\right) \subset \cdots \subset\left(V_{m}, W_{n}\right) \subset \cdots
$$

such that

(1) each $\left(V_{n}, W_{n}\right)$ is torsion-closed in $(V, W)$ and is a finite direct $\operatorname{sum}\left(V_{n}, W_{n}\right)=$ $\sum_{j=1}^{r_{n}}\left(V_{n j}, W_{n j}\right)$ with $\left(V_{n j}, W_{n j}\right)$ of type $\operatorname{III}^{m_{n j}}$ and $\left(\min \left\{m_{n j}: 1<j<r_{n}\right\}\right)_{n=1}^{\infty}$ unbounded.

Proof. Since $(V, W)$ is nonzero and torsion-free, there exists a nonzero $w$ in $W$. Put $\left(V_{1}, W_{1}\right)=\operatorname{tc}_{(V, W)}\left(\varnothing,\left\{w_{1}\right\}\right)$. By Theorem 1 of [2], if $\left(V_{1}, W_{1}\right)$ were infinite-dimensional, it would be a nonzero pure subsystem of $(V, W)$ of rank 1 , contradicting the assumption on $(V, W)$. Hence $\left(V_{1}, W_{1}\right)$ is a torsion-closed subsystem of type III $^{m_{11}}$, with $m_{11} \geqslant 1$.

For $n \geqslant 1$ suppose that there exists a sequence of finite-dimensional torsionclosed subsystems $\left(V_{1}, W_{1}\right) \subset \cdots \subset\left(V_{n}, W_{n}\right)$, where, with the decompositions as in (1),

$$
\min \left\{m_{k j}: 1 \leqslant j \leqslant r_{k}\right\} \geqslant k \text { for } k=1, \ldots, n .
$$

By the assumption on $(V, W)$, it has no direct summand of type III $^{m}$ for any positive integer $m$. Therefore, by Theorem 2 of [2], $\left(V_{n}, W_{n}\right)$ is contained in a subsystem $(U, Z)$ of $(V, W)$ which is a finite direct $\operatorname{sum}(U, Z)=\sum_{j=1}^{s} \cdot\left(U_{j}, Z_{j}\right)$ with $\left(U_{j}, Z_{j}\right)$ of type $\operatorname{III}^{p_{j}}$ and $\min \left\{p_{j}: 1 \leqslant j \leqslant s\right\} \geqslant n+1$. Put $\left(V_{n+1}, W_{n+1}\right)=$ $\operatorname{tc}_{(V, W)}(U, Z)$. We have $\operatorname{rank}(U, Z)=s$. If $\left\{z_{j}\right\}_{j=1}^{s}$ is a subset of $Z$ such that

Received by the editors August 22, 1977 and, in revised form, June 9, 1978 and July 25, 1979.

AMS (MOS) subject classifications (1970). Primary 15A21, 16A64; Secondary 16A46.

Key words and phrases. System, purely simple, rank. 


$$
(U, Z)=\operatorname{tc}_{(U, Z)}\left(\varnothing,\left\{z_{j}\right\}_{j-1}^{s}\right),
$$

then

$$
\left(V_{n+1}, W_{n+1}\right)=\operatorname{tc}_{(V, W)}\left(\varnothing,\left\{z_{j}\right\}_{j=1}^{s}\right) .
$$

Therefore if $r_{n+1}=\operatorname{rank}\left(V_{n+1}, W_{n+1}\right)$, then $r_{n+1}<s$. Since by assumption $\left(V_{n+1}, W_{n+1}\right)$ is not pure in $(V, W)$, it follows from [2, Theorem 1] that $\left(V_{n+1}, W_{n+1}\right)$ has a direct summand $\left(V_{n+1,1}, W_{n+1,1}\right)$ of some type III $^{m_{n+1,1}}$. Since the rank of a direct complement of $\left(V_{n+1,1}, W_{n+1,1}\right)$ in $\left(V_{n+1}, W_{n+1}\right)$ is $r_{n+1}-1$, it follows by induction on $r_{n+1}$ that $\left(V_{n+1}, W_{n+1}\right)$ is a direct sum,

$$
\left(V_{n+1}, W_{n+1}\right)=\sum_{j=1}^{r_{n+1}}\left(V_{n+1, j}, W_{n+1, j}\right)
$$

with $\left(V_{n+1, j}, W_{n+1, j}\right)$ of type III ${ }^{m_{n+1 j}}$.

To complete the inductive existence proof of the required sequence of subsystems, it suffices to show that $\min \left\{m_{n+1, j}: 1 \leqslant j \leqslant r_{n+1}\right\} \geqslant \min \left\{p_{j}: 1<j<s\right\}$. Suppose to the contrary that $\left(V_{n+1}, W_{n+1}\right)$ has a direct decomposition

$$
\left(V_{n+1}, W_{n+1}\right)=\left(X_{1}, Y_{1}\right) \dot{+}\left(X_{2}, Y_{2}\right)
$$

with $\left(X_{1}, Y_{1}\right)$ of type III ${ }^{m}$ and $m<p_{j}$ for all $j=1, \ldots, s$. Let $(\pi, \rho)$ be the projection of $\left(V_{n+1}, W_{n+1}\right)$ onto $\left(X_{1}, Y_{1}\right)$ with kernel $\left(X_{2}, Y_{2}\right)$, and for $j=$ $1, \ldots, s$, let $\Gamma_{j}$ be a chain of type $\operatorname{III}^{p_{j}}$ spanning $\left(U_{j}, Z_{j}\right)$. Then by [1, Theorem 6.3(c)], $(\pi, \rho) \Gamma_{j}=0$. Hence each $\left(U_{j}, Z_{j}\right)$ and therefore also $(U, Z)$ is contained in $\left(X_{2}, Y_{2}\right)$. Since $\left(X_{2}, Y_{2}\right)$ is torsion-closed in $\left(V_{n+1}, W_{n+1}\right)$, which in turn is torsionclosed in $(V, W),\left(X_{2}, Y_{2}\right)$ is torsion-closed in $(V, W)$. Hence $\left(V_{n+1}, W_{n+1}\right)=$ $\operatorname{tc}_{(V, W)}(U, Z) \subset\left(X_{2}, Y_{2}\right)$, a contradiction.

THEOREM 1. Every nonzero system $(V, W)$ has a nonzero pure subsystem of rank $<\aleph_{0}$. Hence a system of rank $>\aleph_{0}$ cannot be purely simple.

Proof. By [1, Proposition 9.12], $t(V, W)$ is pure in $(V, W)$. So if $t(V, W) \neq 0$, $(V, W)$ has a nonzero pure subsystem of rank 0 . Thus we may assume that $(V, W)$ is torsion-free. If $(V, W)$ has a nonzero pure subsystem of finite rank, we are done. Otherwise, put $(X, Y)=\cup_{n=1}^{\infty}\left(V_{n}, W_{n}\right)$, where the sequence $\left(\left(V_{n}, W_{n}\right)\right)_{n-1}^{\infty}$ is as described in the Lemma. As a union of an ascending sequence of torsion-closed subsystems of $(V, W),(X, Y)$ is torsion-closed in $(V, W)$. Suppose that $(X, Y)$ has a direct summand $(U, Z)$ of type III ${ }^{m}$. Then for all $n$ beyond some $n_{0},(U, Z)$ is contained in $\left(V_{n}, W_{n}\right)$, and hence is a direct summand of $\left(V_{n}, W_{n}\right)$. Since any two decompositions of $\left(V_{n}, W_{n}\right)$ into a direct sum of indecomposable subsystems are isomorphic (see [1, p. 309]), this would imply that $\min \left\{m_{n j}: 1<j<r_{n}\right\}<m$ for all $n>n_{0}$, contradicting the unboundedness condition. Hence $(X, Y)$ contains no such $(U, Z)$, and by [2, Theorem 1], $(X, Y)$ is pure in $(V, W)$. By construction $\cup_{n=1}^{\infty} W_{n}$ has a linear countable basis $\left\{w_{k}\right\}$. Since $(X, Y)=\operatorname{tc}_{(X, Y)}\left(\varnothing,\left\{w_{k}\right\}\right)$, a subset $L$ of this basis is a basis of $(X, Y)$ with respect to generation. Since $(V, W)$ has no nonzero pure subsystem of finite rank, $L$ is infinite and rank $(X, Y)=\aleph_{0}$. 
THEOREM 2. A torsion-free system is purely simple of rank $\aleph_{0}$ if and only if

(a) $(V, W)$ is the union of an ascending sequence of subsystems $\left(V_{1}, W_{1}\right) \subset$ $\left(V_{2}, W_{2}\right) \subset \cdots$ satisfying (1) and

(b) every nonzero proper torsion-closed subsystem of $(V, W)$ has a direct summand of type III $^{m}$ for some positive integer $m$.

Proof. If $(V, W)$ is purely simple of rank $\boldsymbol{\aleph}_{0}$, it has no nonzero pure subsystem of finite rank. Hence, as in the proof of Theorem $1,(V, W)$ contains a nonzero pure subsystem $(X, Y)=\cup_{n=1}^{\infty}\left(V_{n}, W_{n}\right)$ where $\left(\left(V_{n}, W_{n}\right)\right)_{n=1}^{\infty}$ is an ascending sequence satisfying (1). Since $(V, W)$ is purely simple, $(X, Y)=(V, W)$ and (a) holds. By [2, Theorem 1], a nonzero torsion-closed subsystem with no direct summand of type III $^{m}$ is pure in $(V, W)$, and hence cannot be proper. This establishes (b).

Conversely, suppose that $(V, W)$ satisfies (a) and (b) and has a nonzero pure subsystem $(S, T) .(S, T)$ is then torsion-closed in $(V, W)$, and so by (b) has a direct summand $(U, Z)$ of type $\operatorname{III}^{m}$. By transitivity of purity $(U, Z)$ is pure in $(V, W)$, hence by [1, Theorem 5.5] it is a direct summand of $(V, W)$. Since $(V, W)$ has the same kind of structure as $(X, Y)$ in the proof of Theorem 1 , such a direct summand $(U, Z)$ is impossible. So $(V, W)$ must be purely simple. As in the proof of Theorem $1, \operatorname{rank}(V, W) \leqslant \aleph_{0}$. If $(V, W)$ were of finite rank, it would be of the form $(V, W)=\operatorname{tc}_{(V, W)}(\varnothing, L)$ where $L$ is a finite subset of $W$. Then $L \subset W_{n_{0}}$ for some $n_{0}$, and since $\left(V_{n_{0}}, W_{n_{0}}\right)$ is torsion-closed, $(V, W) \subset\left(V_{n_{0}}, W_{n_{0}}\right)$. However, by the unboundedness condition the sequence of (a) cannot be stationary. This contradiction shows that $\operatorname{rank}(V, W)=\aleph_{0}$.

ACKNOWLEDGEMENT. It is with pleasure that $I$ thank the referee not only for pointing out an error in an earlier draft of this paper but also for making extensive suggestion for the improvement of the final draft.

\section{REFERENCES}

1. N. Aronszajn and U. Fixman, Algebraic spectral problems, Studia Math. 30 (1968), 273-338.

2. U. Fixman and F. Zorzitto, A purity criterion for pairs of linear transformations, Canad. J. Math. 26 (1974), 734-745.

3. F. Okoh, $A$ bound on the rank of purely simple systems, Trans. Amer. Math. Soc. 232 (1977), 169-186.

Department of MATHematics, University OF Nigeria, NsUKKa, Nigeria

Current address: 11 Kellythorne Drive, Don Mills, Ontario, Canada M3A 2L5 